SLAC-PUB-11017

January 2005

\title{
Performance of the BABAR-DIRC*
}

\author{
Jochen Schwiening \\ Stanford Linear Accelerator Center, Stanford University, Stanford, CA 94309 \\ Representing the BABAR-DIRC Collaboration ${ }^{\ddagger}$
}

\begin{abstract}
A new type of ring-imaging Cherenkov detector is being used for hadronic particle identification in the $B_{A} B A R$ experiment at the SLAC B Factory (PEP-II). This detector is called DIRC, an acronym for Detection of Internally Reflected Cherenkov (Light). This paper describes the performance of the DIRC during the first 5 years of operation.
\end{abstract}

Talk presented at 5th Workshop On RICH Detectors (RICH04)

Playa del Carmen, Mexico

Nov 30-Dec 05, 2004

${ }^{*}$ Work supported by Department of Energy contract DE-AC02-76SF00515 (SLAC), DE-AC03-76SF00098 (LBNL), DE-AM03-76SF0010 (UCSB), and DE-FG03-93ER40788 (CSU); the National Science Foundation grant PHY-95-11999 (Cincinnati). 


\section{¥ The BABAR-DIRC Collaboration}

R. Aleksan, ${ }^{2}$ D. Arnaud,${ }^{4}$ D. Aston, ${ }^{1}$ N. van Bakel,${ }^{1}$ D. Bernard,${ }^{5}$ P. Bourgeois, ${ }^{2}$ F. Brochard,${ }^{5}$ D.N. Brown,${ }^{6}$ J. Chauveau, ${ }^{3}$ M. Convery, ${ }^{1}$ S. Emery, ${ }^{2}$ A. Gaidot,${ }^{2}$ X. Giroux,${ }^{4}$ Ph. Grenier,${ }^{5}$ G. Grosdidier,${ }^{4}$ T. Hadig, ${ }^{1}$

G. Hamel de Monchenault, ${ }^{2}$ B. Hartfiel, ${ }^{9}$ A. Hoecker, ${ }^{4}$ M. John, ${ }^{3}$ R.W. Kadel, ${ }^{6}$ M. Legendre, ${ }^{2}$ J. Libby, ${ }^{1}$ A.-M. Lutz, ${ }^{4}$ J. Malcles,${ }^{3}$ G. Mancinelli, ${ }^{8}$ B.T. Meadows, ${ }^{8}$ K. Mishra,${ }^{8}$ D. Muller, ${ }^{1}$ J. Ocariz,${ }^{3}$ M. Pripstein, ${ }^{6}$ B.N. Ratcliff, ${ }^{1}$ L. Roos,${ }^{3}$ S. Schrenk, ${ }^{5}$ M.-H. Schune, ${ }^{4}$ J. Schwiening, ${ }^{1}$ V. Shelkov, ${ }^{6}$ M.D. Sokoloff, ${ }^{8}$ S. Spanier,${ }^{10}$ K. Suzuki, ${ }^{1}$ G. Therin,${ }^{3}$ Ch. Thiebaux,${ }^{5}$ G. Vasileiadis, ${ }^{5}$ G. Vasseur, ${ }^{2}$ J. Va'vra, ${ }^{1}$ R.J. Wilson, ${ }^{7}$ G. Wormser, ${ }^{4}$ A. Yarritu, ${ }^{1}$ Q. Zeng, ${ }^{8}$ M. Zito. ${ }^{2}$

${ }^{1}$ Stanford Linear Accelerator Center, Stanford, CA 94309, USA.

${ }^{2}$ CEA, DAPNIA, CE-Saclay, F-91191, Gif-sur-Yvette Cedex, France.

${ }^{3}$ LPNHE des Universités Paris 6 et Paris 7, Tour 33, Bc 200, 4 Place Jussieu, F-75252, Paris, Cedex 05, France.

${ }^{4}$ LAL Orsay, Universite Paris Sud, Batiment 200, F-91405 Orsay Cedex, France.

${ }^{5}$ Ecole Polytechnique, Laboratoire Leprince-Ringuet, Route de Saclay, F-91128 Palaiseau Cedex, France.

${ }^{6}$ Lawrence Berkeley National Laboratory, One Cyclotron Road, Berkeley, CA 94720, USA.

${ }^{7}$ Dept. of Physics, Colorado State University, Fort Collins, CO 80523, USA. ${ }^{8}$ Dept. of Physics, University of Cincinnati, Cincinnati, OH 45221, USA. ${ }^{9}$ Dept.of Physics, University of California, Los Angeles, CA 90095, USA. ${ }^{10}$ Dept. of Physics, University of Tennessee, Tennessee, TN 37996, USA. 


\section{Introduction}

The Particle Identification (PID) system being used in $B A B A R$ [1] is a new kind of ringimaging Cherenkov detector called the DIRC [2] (the acronym DIRC stands for Detector of Internally Reflected Cherenkov light). It is designed to be able to provide excellent $\pi / K$ separation for all tracks from $B$-meson decays from the pion Cherenkov threshold up to 4.2 $\mathrm{GeV} / \mathrm{c}$. PID below $700 \mathrm{MeV} / \mathrm{c}$ exploits also the $d E / d x$ measurements in the silicon vertex tracker and drift chamber.

The DIRC principle, design, and construction are described in detail in Ref. [3]. Briefly, the Dirc uses $4.9 \mathrm{~m}$ long, rectangular bars made from synthetic fused silica as Cherenkov radiator and light guide. A charged particle with velocity $v$, traversing the fused silica bar with index of refraction $n(\sim 1.473)$, generates a cone of Cherenkov photons of half-angle $\theta_{C}$ with respect to the particle direction, where $\cos \theta_{C}=1 / \beta n(\beta=v / c, c=$ velocity of light). For particles with $\beta \approx 1$, some photons always lie within the total internal reflection limit, and are transported efficiently to either one or both ends of the bar, depending on the particle incident angle. Since the bar has a rectangular cross section and is made to optical precision, the magnitude of the Cherenkov angle is conserved during the reflection at the radiator bar surfaces. The photons are imaged via "pin-hole" focussing by expanding through a standoff region filled with 6000 litres of purified water onto an array of 10752 densely packed photomultiplier tubes placed at a distance of about $1.2 \mathrm{~m}$ from the bar end. Imaging in the $B A B A R$ DIRC occurs in three dimensions, by recording the location and the time at which a given PMT is hit. The expected single photon Cherenkov angle resolution is about $9 \mathrm{mrad}$, dominated by a geometric term that is due to the sizes of bars, PMTs and the expansion region, and a chromatic term from the photon production. The accuracy of the time measurement is limited by the intrinsic $1.5 \mathrm{~ns}$ transit time spread of the PMTs.

\section{Operational Issues}

Soon after the DIRC was fully commissioned in late 1999, it achieved performance close to that expected from Monte Carlo simulation and has been running efficiently and reliably ever since. The system has been robust and stable, and, indeed, serves also as a background detector for PEP-II tuning. After five years of running, nearly $99 \%$ of PMTs and electronic channels are operating with nominal performance. Details of the DIRC operational experience are described in Ref. [3]. This section describes two issues: the background in the DiRC and the PMT corrosion.

The background in the DIRC is dominated by low energy photons from the PEP-II machine hitting the water-filled standoff box. The time-to-digital converter (TDC) chip used originally in the DIRC data readout was designed such that a dead time of about $5 \%$ occurs at an input rate of $250 \mathrm{kHz}$. Some care in machine tuning is required to stay under a limit of $250 \mathrm{kHz} /$ tube. To monitor that rate, one PMT in each sector is read out via a scaler. In early 2000, at a peak luminosity value that corresponds to only one third of the design luminosity, the PMT rates reached a level that caused noticeable dead times. Due to those findings, lead shielding was installed for the DIRC. Since January 2001 an engineered, 


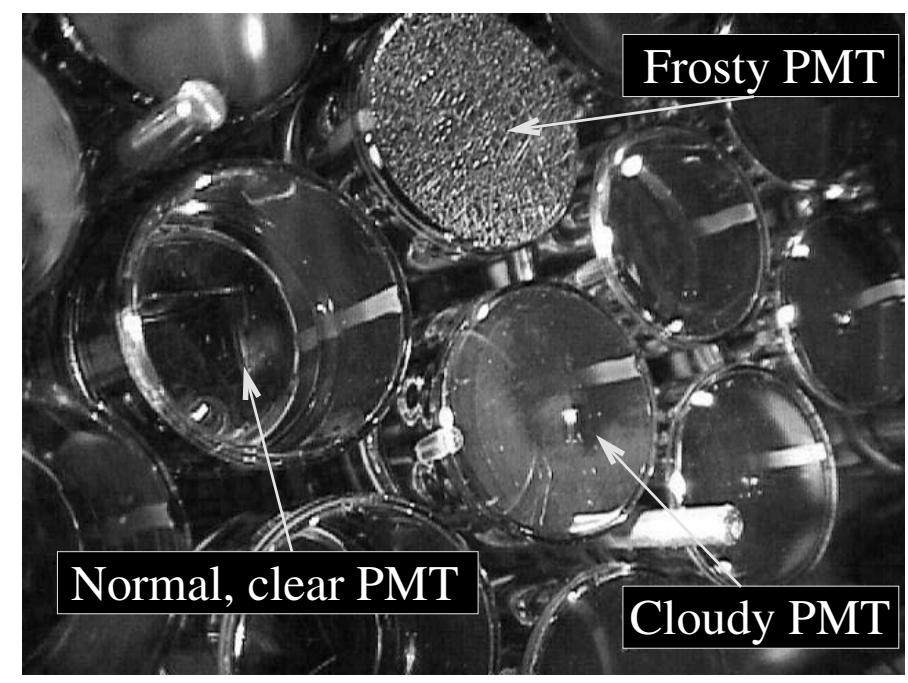

Figure 1: Example of PMT corrosion observed in October 1999, when the water was drained from the standoff box.

homogeneous lead shielding of $5 \mathrm{~cm}$ to $8 \mathrm{~cm}$ thickness has covered the inside radius of the standoff box. This shielding keeps the scaler rates well below the $200 \mathrm{kHz} / \mathrm{tube}$ level even at luminosities that significantly exceed the design luminosity.

During the shutdown that followed the 2001-2002 run, the TDCs were replaced with a faster version with deeper buffering capable of accepting a $2.5 \mathrm{MHz}$ input rate with less than $5 \%$ dead time. Based on the projected increase in PEP-II luminosity through 2010 the new TDCs are not expected to suffer from any significant dead time due to accelerator induced background during the lifetime of the experiment.

Some deterioration of the PMT front glass windows (made of B53 Borosilicate glass) that are immersed in the ultra-pure water of the standoff box has been observed since 2000, as shown in Figure 1. With water in the standoff box, these features are much less noticeable as water provides good optical coupling even to corroded glass. For most of the tubes, the observable effect is typically a slight cloudiness, but for about 50 tubes, it is much more pronounced and leads to so-called frosty PMTs. Extensive R\&D has demonstrated that the effect is associated with a loss of sodium and boron from the surface of the glass. For most tubes, the leaching rate is a few microns per year, and is expected to be acceptable for the full projected ten year lifetime of the experiment. However, for the $\sim 50$ tubes, the incorrect glass was used by the manufacturer. That glass does not contain zinc, making it much more susceptible to rapid leaching. This leaching may eventually lead to either a loss of performance, or some risk of mechanical failure of the face plates for these tubes. R\&D tests show that the mechanical failure of a PMT does not induce mechanical failure in neighboring PMTs.

Loss of photon detection efficiency can arise from this corrosion of PMT front glass windows as well as from possible deterioration of the water transparency or pollution of bar or window surfaces. Direct measurements of the number of Cherenkov photons observed in di-muon events as a function of time can be used to determine any degradation of the photon 
yield. An analysis using di-muon events from late 1999 through July 2004 shows a photon loss of 1-2 \%/year. There is no significant dependence of the loss rate on the radiator bar number, the position of track long the bar length, or the location of the Cherenkov ring in the PMT plane.

The reason for the loss in photon yield is not fully understood. The observed PMT glass corrosion, resulting in "frosty" tubes, affects too small a number of them to be a significant effect. R\&D on photocathode aging, where DIRC PMT photocathodes are explosed to extremely large doses of UV light, rule this mechanism out as explanation for the photon loss. Dynode aging, on the other hand, is one possible source as the DIRC PMTs have accumulated at least 30-40 Coulombs per anode in the five years of operation. ADC calibrations in 2003 showed a loss of the PMT gain that is consistent with expected dynode aging. However, the lower PMT gain only accounts for about $30 \%$ of the observed loss in photon yield. Operationally, the PMT gain was recovered in 2003 by raising the high-voltage of the DIRC PMTs by an average of 43V. Nevertheless, even if the deterioration continues at the rate of $1.5 \%$ year, the impact on the particle identification power of the DIRC is small. For instance, after 10 years the $\pi / \mathrm{K}$ separation at $3.5 \mathrm{GeV} / \mathrm{c}$ momentum would go from the current value of 3.5 standard deviations to 3.2 standard deviations.

\section{Results}

Since the inclusive branching fraction $B^{0} \rightarrow K^{+} X$ is about $78 \%$ [4], $\mathrm{K} / \pi$ separation is essential for the physics program of $B A B A R$. The DIRC plays a central role in final state selection and $B$ meson flavor tagging for CP measurements. This section provides an overview of the physics performance. A more detailed discussion of the use of DIRC in BABAR physics analyses can be found in Ref. [3].

In the absence of correlated errors, the resolution $\left(\sigma_{C, t r a c k}\right)$ on the track Cherenkov angle should behave as:

$$
\sigma_{C, \text { track }}^{2}=\sigma_{C, \gamma}^{2} / N_{\gamma}+\sigma_{\text {track }}^{2},
$$

where $N_{\gamma}$ is the number of detected photoelectrons, $\sigma_{C, \gamma}$ is the single photon Cherenkov angle resolution and $\sigma_{\text {track }}$ is the uncertainty of the track direction in the DiRC. Figure 2(a) shows the single photon angular resolution $\Delta \theta_{C, \gamma}$ obtained from di-muon events. There is a broad background of less than $10 \%$ relative height under the peak that originates mostly from track-associated sources, such as $\delta$ rays, reflections off the glue-fused silica boundaries, and combinatorial background. The width of the peak translates to a resolution of about $9.6 \mathrm{mr}$, in good agreement with the expected value. The measured time resolution, shown in Figure 2(b), is $1.7 \mathrm{~ns}$, close to the intrinsic $1.5 \mathrm{~ns}$ transit time spread of the PMTs.

The average value of $N_{\gamma}$, shown in Figure 3, varies between about 17 for tracks with nearly perpendicular incidence to nearly 60 for polar angles towards the forward and backward regions. The increase in the number of photons for tracks in the forward direction compensates for the reduced average separation in the Cherenkov angle for different particle hypotheses due to the increased track momenta in this region. 

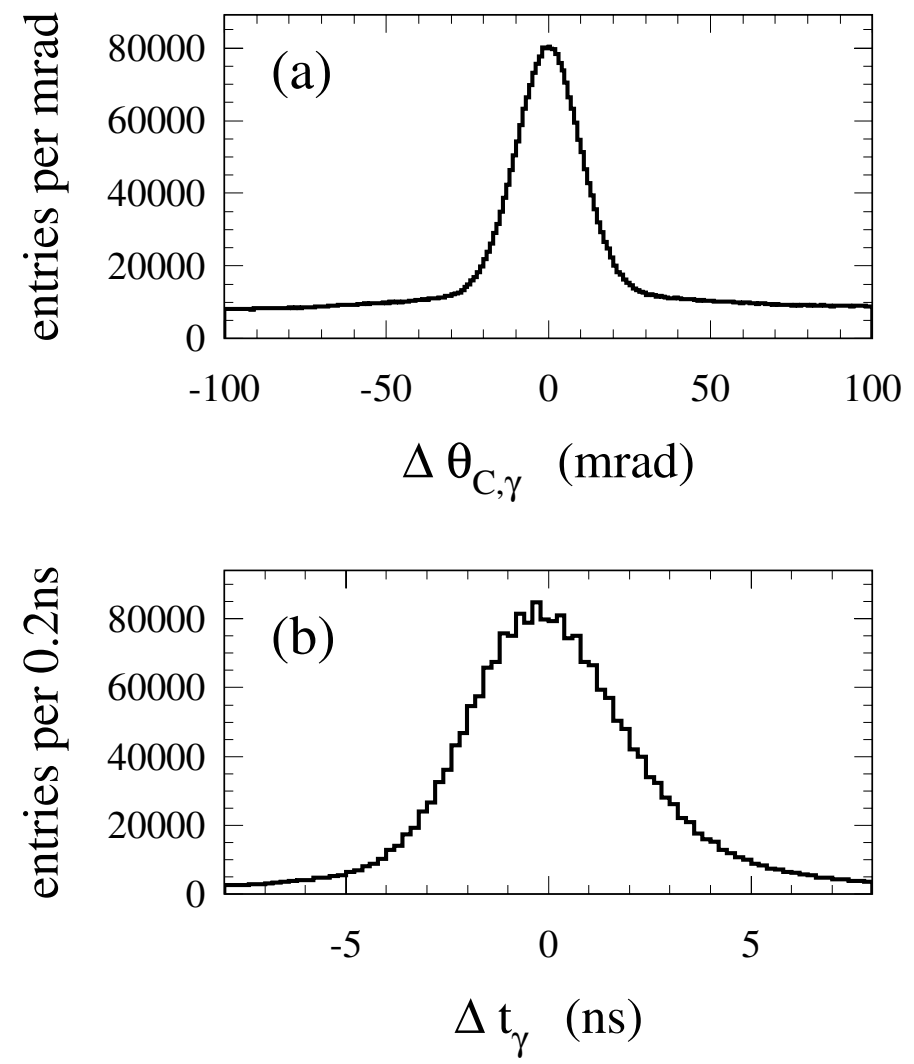

Figure 2: The difference between (a) the measured and expected Cherenkov angle for single photons, $\Delta \theta_{C, \gamma}$, and (b) the measured and expected photon arrival time, for single muons in $\mu^{+} \mu^{-}$events. 


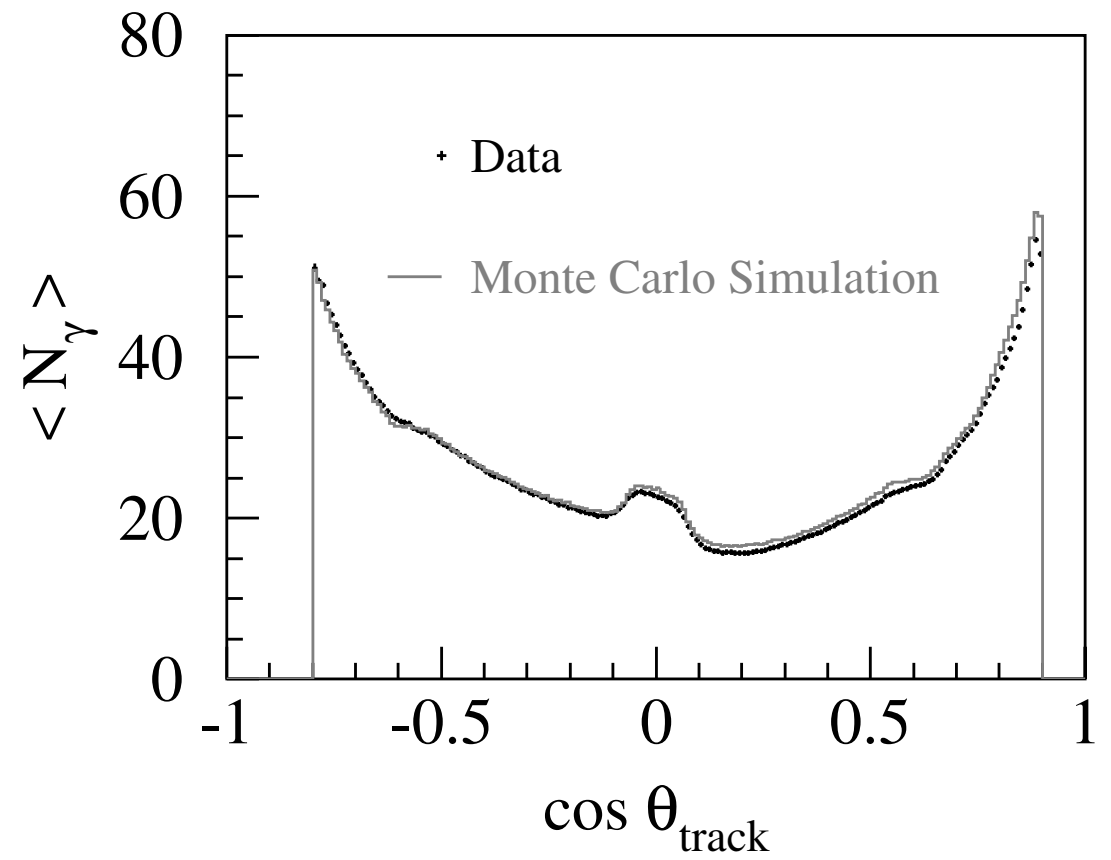

Figure 3: Number of detected photons versus track polar angle for reconstructed tracks in di-muon events compared to Monte Carlo simulation. The mean number of photons in the simulation has been tuned to match the data.

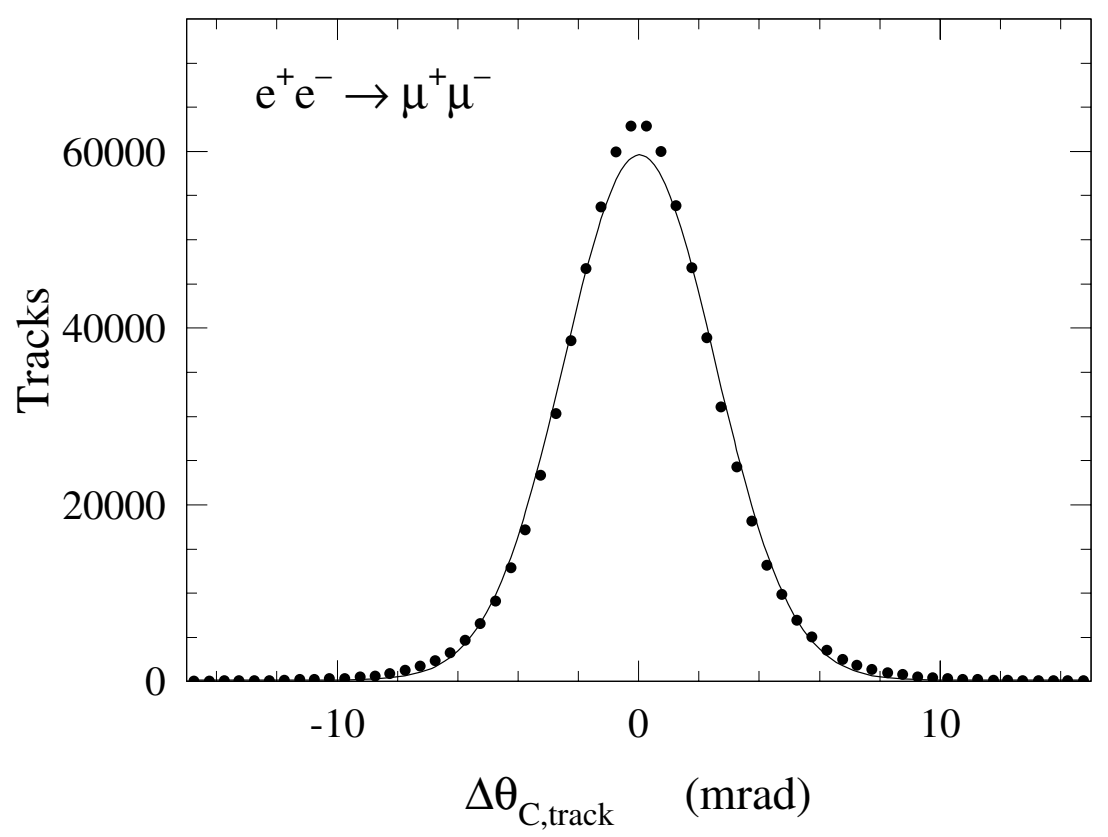

Figure 4: Resolution of the reconstructed Cherenkov polar angle per track for di-muon events. The curve shows the result of a Gaussian fit with a resolution of $2.4 \mathrm{mr}$. 
The Cherenkov angle resolution, $\sigma_{C, \text { track }}$, for tracks from di-muon events, $e^{+} e^{-} \rightarrow \mu^{+} \mu^{-}$, is shown in in Figure 4. The width assuming a single Gaussian distribution is $2.5 \mathrm{mr}$. The resolution is $14 \%$ larger than the design goal of $2.2 \mathrm{mr}$, which was estimated from the extensive study of a variety of prototypes, including a beam test.

The $D^{*+} \rightarrow \pi^{+}\left(D^{0} \rightarrow K^{-} \pi^{+}\right)$decay chain ${ }^{\dagger}$ is well suited to probe the pion and kaon identification capabilities of the DIRC. It is kinematically well constrained and the momentum spectrum of the charged pions and kaons covers the range accessible by $B$ meson decay products in $B_{A} B_{A R}$. Figure 5 shows the $K^{-} \pi^{+}$invariant-mass spectrum from inclusive $D^{*}$ production. Both tracks are within the DIRC acceptance and at least 10 Cherenkov photons are required for the charged pion hypothesis. The lower peak in Figure 5 shows the same sample after one track is identified as a kaon using the likelihood ratio from the DIRC.

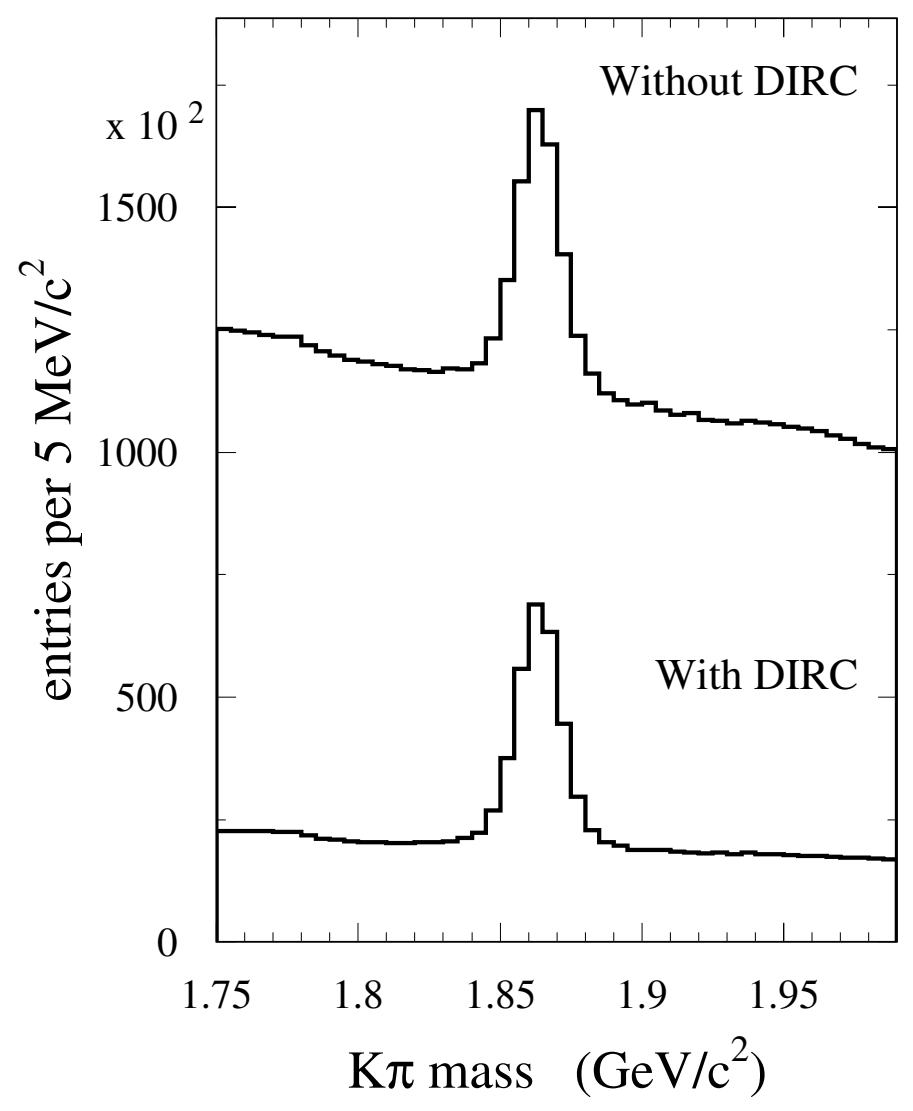

Figure 5: Invariant $\mathrm{K}^{+} \pi^{-}$mass spectrum for kinematically reconstructed $D^{0}$ mesons in the decay $D^{*} \rightarrow D^{0} \pi$ as described in the text, without (top) and with (bottom) the use of the DiRC to identify the kaon.

\footnotetext{
${ }^{\dagger}$ Unless explicitly stated, charge conjugate decay modes are assumed throughout this section.
} 


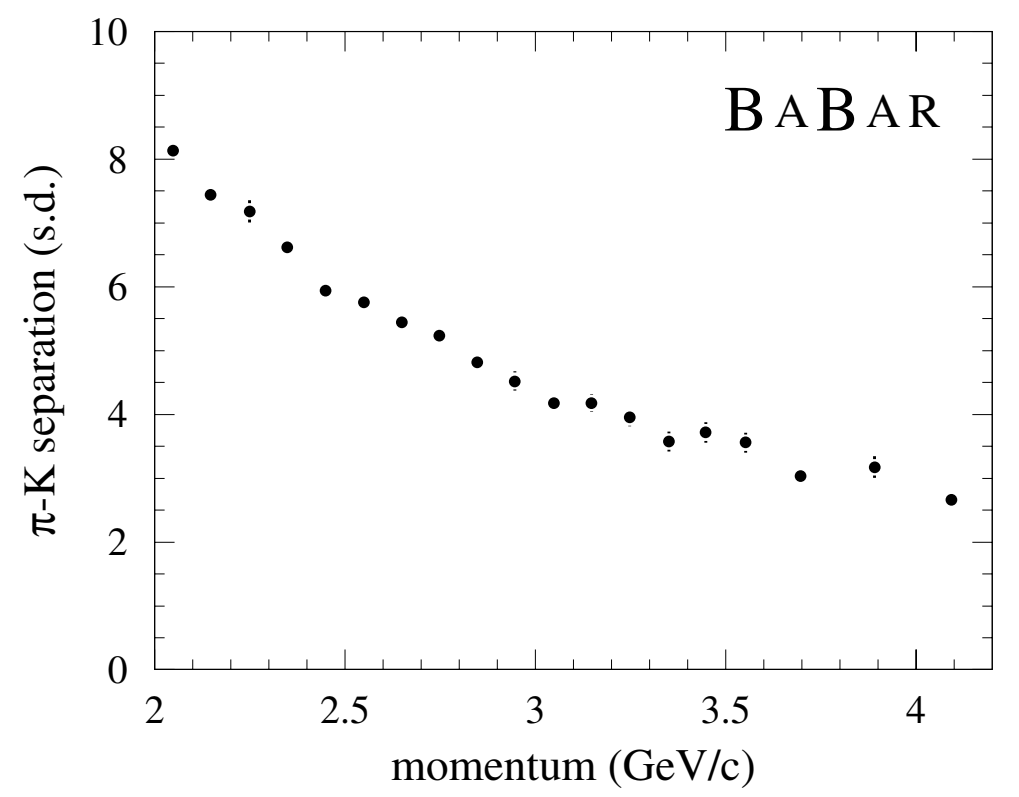

Figure 6: DiRC $\pi$-K separation versus track momentum measured in $D^{0} \rightarrow K^{-} \pi^{+}$decays selected kinematically from inclusive $D^{*}$ production.

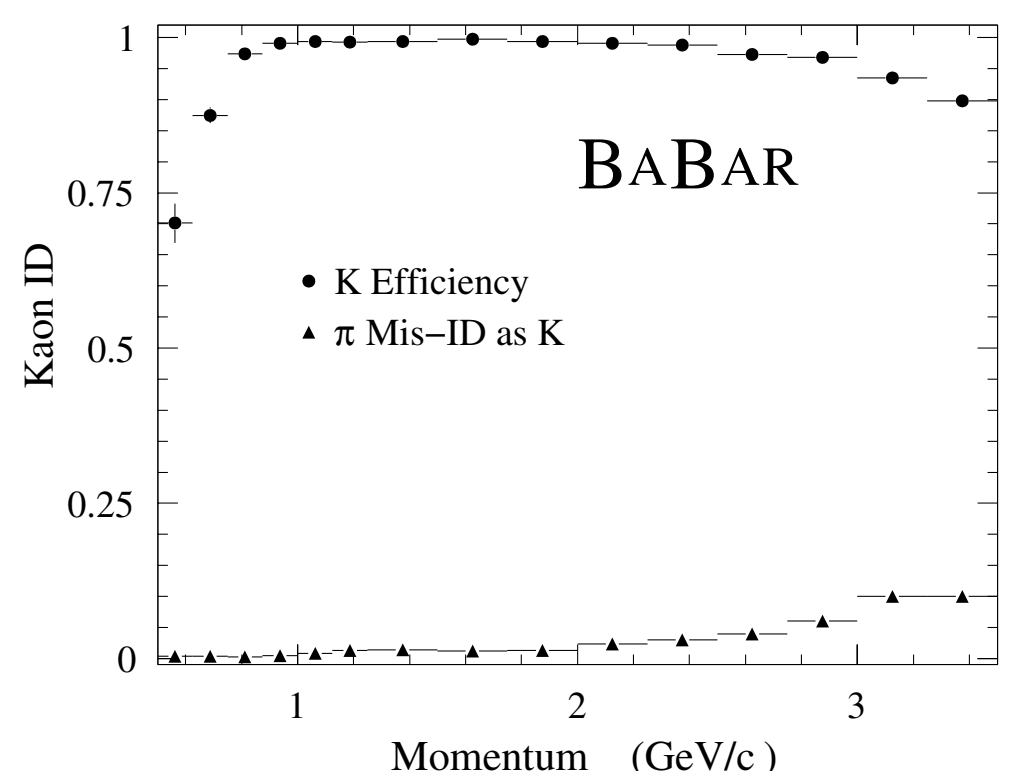

Figure 7: Efficiency and misidentification probability for the selection of charged kaons as a function of track momentum, for a particular choice of particle selection criteria. The data use $D^{0} \rightarrow K^{-} \pi^{+}$decays selected kinematically from inclusive $D^{*}$ production. 
The pion-kaon separation power is defined as the difference of the mean Cherenkov angles for pions and kaons assuming a Gaussian-like distribution, divided by the measured track Cherenkov angle resolution. As shown in Figure 6, the separation between kaons and pions is about $4 \sigma$ at $3 \mathrm{GeV} / \mathrm{c}$ declining to about $2.5 \sigma$ at $4.2 \mathrm{GeV} / \mathrm{c}$.

The efficiency for correctly identifying a charged kaon that traverses a radiator bar and the probability to wrongly identify a pion as a kaon are also determined from the inclusive $D^{*}$ sample and are shown as a function of the track momentum in Figure 7 for a particular choice of particle selection criteria. The kaon selection efficiency and pion misidentification, integrated over the $K$ and $\pi$ momentum spectra of the $D^{*}$ control sample, are $97.97 \pm 0.07 \%$ (stat. only) and $1.83 \pm 0.06 \%$ (stat. only), respectively.

\section{Summary}

The DIRC is a novel ring-imaging Cherenkov detector that is very well-matched to the hadronic particle identification requirements of $B_{A} B A R$. The detector performance achieved is excellent and close to that predicted by the Monte Carlo simulations. The Dirc is robust, stable, and easy to operate. After more than four years of colliding beam data taking, about $99 \%$ of all PMTs and electronic channels are still operating with nominal performance. Additional shielding in the SOB tunnel region was required to reduce the sensitivity to beam-induced backgrounds. To allow operation at luminosities up to $2 \times 10^{34} / \mathrm{cm}^{2} \sec (\approx 7$ times the original PEP-II design luminosity) that may occur during the next few years, faster front-end cards and faster TDCs with deeper buffering have been installed.

\section{References}

[1] B. Aubert et al., "The BABAR detector," Nucl. Instr. Methods A479 (2002) pp. 1-116.

[2] B. N. Ratcliff, "The B factory detector for PEP-II: a status report," SLAC-PUB-5946 (1992); B. N. Ratcliff, "The DIRC counter: a new type of particle identification device for B factories," SLAC-PUB-6047 (1993); P. Coyle et al., "The DirC counter: a new type of particle identification device for B factories," Nucl. Instr. Methods A343 (1994) pp. 292-299.

[3] I. Adam et al., " The DiRC Particle Identification System for the BABAR Experiment," Nucl. Instr. Methods A538 (2005) pp. 281-357.

[4] Particle Data Group, K. Hagiwara et al., Phys.Rev. D66, 010001-1 (2002). 